
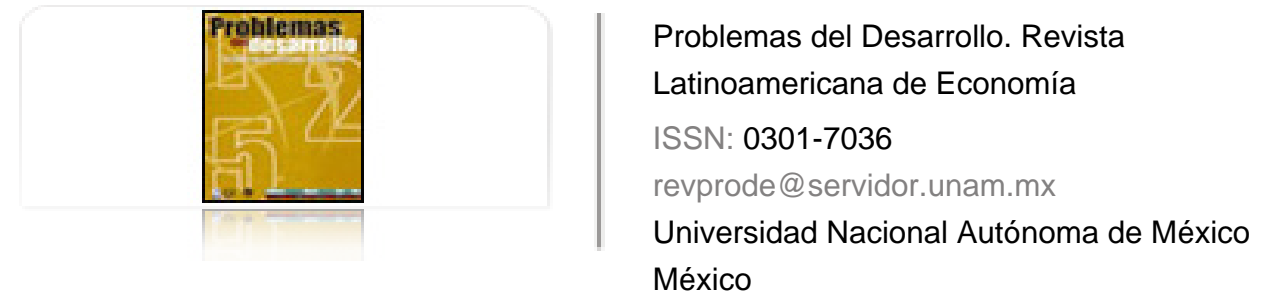

Mungaray, Alejandro; Ramírez, Natanael; Aguilar, José G.; Beltrán, José M.

Poder de mercado en microempresas de Baja California

Problemas del Desarrollo. Revista Latinoamericana de Economía, vol. 38, núm. 148, enero-marzo, 2007, pp. 173-194

Universidad Nacional Autónoma de México

Distrito Federal, México

Disponible en: http://www.redalyc.org/articulo.oa?id=11820155008

Cómo citar el artículo

- Número completo

- Más información del artículo

Página de la revista en redalyc.org

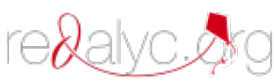

Sistema de Información Científica

Red de Revistas Científicas de América Latina, el Caribe, España y Portugal Proyecto académico sin fines de lucro, desarrollado bajo la iniciativa de acceso abierto 


\section{Poder de mercado en microempresas de Baja California}

\section{Alejandro Mungaray* Natanael Ramírez** José G. Aguilar*** José M. Beltrán ${ }^{* * * *}$}

Fecha de recepción: 5 de julio de 2006. Fecha de aceptación: 26 de septiembre de 2006.

\section{Resumen}

El presente trabajo analiza la posibilidad de que algunos tipos de microempresas puedan actuar con poder de mercado, pese a la marginación socioeconómica en la que operan. Al concentrarse en zonas carentes de infraestructura y con rasgos de marginación, esas empresas se ven con posibilidades de fijar un precio mayor al del mercado, actuando en un marco de competencia distinto al de competencia perfecta. El consumidor, por su parte, está dispuesto a pagar un precio más elevado por el mismo bien, debido a lo alto de los costos (en términos de tiempo y esfuerzo) que trae consigo el adquirirlo en zonas más comerciales. Este trabajo se fundamenta en un análisis de 29 microempresas, en el cual los resultados muestran una relación positiva entre el indice de Lerner y el nivel de marginación de las colonias donde se concentran.

Palabras clave: poder de mercado, microempresas, marginación socioeconómica, competencia, indice de Lerner.

* Profesor de tiempo completo en la Facultad de Economía y Relaciones Internacionales de la Universidad Autónoma de Baja California. Además es integrante del Sistema Nacional de Investigadores, nivel I. Correo electrónico: mungaray@uabc.mx

* Profesor de tiempo completo de la Facultad de Economía y Relaciones Internacionales de la Universidad Autónoma de Baja California. Además es integrante del Sistema Nacional de Investigadores, nivel c. Correo electrónico: natanael@uabc.mx

*** Profesor de tiempo completo de la Facultad de Economía y Relaciones Internacionales de la Universidad Autónoma de Baja California. Correo electrónico: gaba@uabc.mx

**** Consultor en la empresa Deloitte \& Touche. Correo electrónico: jlopezbetran@deloittemx.com 
Abstract

This paper analyzes the possibility that some kinds of micro-companies may have market power, despite the socio-economic marginalization in which they operate. Concentrating on zones lacking infrastructure with a marginalization character, these companies are seen to have the possibility of setting prices higher than the market, acting within a framework of competition that is distinct from perfect competition. The consumer, for his part, is disposed to pay a higher price for the same good, due to the high level of costs (in terms of time and effort) that would be involved in acquiring it in more commercially-developed zones. The study is based on an analysis of 29 micro-companies, the results of which show a positive relationship between the Lerner Index and the level of marginalization in the districts where they are concentrated.

Key words: market power, micro-companies, socio-economic marginalization, competition, Lerner's Index.

\section{Résumé}

Ce travail analyse la possibilité de que certains types de micro entreprises agissent comme pouvoir de marché, malgré la marginalisation socio-économique dans laquelle elles opèrent. En se concentrant dans des zones manquant d'infrastructure qui présentent des aspects de marginalisation, ces entreprises semblent pouvoir fixer un prix supérieur à celui du marché, agissant dans un cadre de compétition distinct à celui de la compétition parfaite. Le consommateur, pour sa part, est disposé à payer un prix plus élevé pour le même bien du fait du surcoût (en termes de temps et d'effort) qu'implique de l'acquérir dans des zones plus commerciales. Ce travail se fonde sur une analyse de 29 micro entreprises, dont les résultats montrent une relation positive entre l'indice de Lerner et le niveau de marginalisation des quartiers où elles se trouvent.

Mots clés: pouvoir de marché, micro entreprises, marginalisation socio-économique, compétition, indice de Lerner.

\section{Resumo}

O presente trabalho analisa a possibilidade de que alguns tipos de microempresas possam agir com poder de mercado, apesar da marginação socio-econômica em que operam. Concentrando-se em zonas carentes de infra-estrutura e com características de marginação, essas empresas encontram-se com possibilidades de fixar um preço mais elevado que o do mercado, agindo em um marco de concorrência distinto ao de concorrência perfeita. O consumidor, por sua parte, está disposto a pagar um preço mais elevado pelo mesmo bem, devido à elevação dos custos (en termos de tempo e esforço) que traz consigo o fato de adquiri-lo en zonas más comerciais. Este trabalho se fundamenta numa análise de 29 microempresas, em que os resultados mostram uma relação positiva entre el índice de Lerner y el nivel de marginación de las colonias donde se concentran.

Palavras chave: poder de mercado, microempresas, marginación socioeconómica, concorrência, indice de Lerner. 


\section{Introducción}

radicionalmente, referirse al poder de mercado ha sido pensar en empresas grandes. Desde la perspectiva de la teoría neoclásica, se ha asumido que la capacidad de limitar la competencia y elevar los precios por encima de los niveles competitivos ( $\mathrm{P}>\mathrm{CMg}$ ) es exclusiva de las empresas de gran tamaño. Esta afirmación se basa en ciertas fortalezas asociadas a esa categoría de empresas, que se acentúa como barrera a la entrada de aquellas de tamaño menor. Entre tales ventajas se encuentran las economías de escala, el control de materia prima en forma de monopsonios u otras de naturaleza análoga, a través de las cuales los grandes productores tienen injerencia en las decisiones sobre el número de oferentes, las cantidades disponibles en el mercado y en los precios del mismo (Blair y Lawrence, 1982). De igual manera, por medio de grandes inversiones en investigación y desarrollo las grandes empresas pueden disponer antes que nadie de la tecnología más sofisticada, o bien, debido a su importancia, logran gestionar mayores oportunidades para obtener ayuda estatal que les garantice la exclusividad de la tecnología más eficiente y de ciertos derechos (Palacio, 2002).

Contrariamente, las posibilidades que la literatura económica le ha concedido a las microempresas ${ }^{1}$ como agentes generadores de poder de mercado son nulas, debido a que ha insistido en analizarlas bajo un marco perfectamente competitivo, donde no existen posibilidades de ejercer algún tipo de presión sobre los oferentes y demandantes. Sin embargo, la falta de un análisis más riguroso de los principales supuestos económicos que sustentan lo anterior — entre ellos la libre entrada y salida de competidores a la industria, el gran número de competidores en el mercado, la disposición igualitaria de tecnología, la existencia de productos homogéneos y de empresas tomadoras de precios en el mercado- limita la capacidad de predicción empírica del modelo neoclásico. Dichos argumentos han sido criticados por

1 Existen varias formas de clasificar a las empresas, ya sea por el número de empleados, el valor de sus activos o de sus ventas. Sin embargo, en este trabajo se considerará microempresa a toda organización económica con fines de lucro que no reporte más de 10 trabajadores, cuyos activos no asciendan a una cantidad mayor de 55000 pesos y que además tenga ventas anuales máximas de 200000 pesos. 
autores como Robinson (1934:104), Schumpeter (1947:149) y Kirzner (1997:60), quienes ponen en duda su veracidad y proponen que se piense en la posibilidad de presencia de poder de mercado. Con este panorama, el presente trabajo tiene como objetivo primordial brindar una estructura de análisis alternativo al modelo de competencia perfecta para entender el comportamiento microempresarial.

La hipótesis de investigación que guía este trabajo considera que la microempresa marginada socioeconómicamente tenga cierto grado de poder de mercado que le asegure su presencia dentro del mismo, debido a que le permite fijar un precio por encima de sus costos marginales. Dicho poder de mercado estaría dado por el grado de diferenciación que le confieren su propia condición de marginación socioeconómica, su capacidad productiva, las habilidades empresariales del dueño y el desempeño laboral de los trabajadores.

La estructura del trabajo es la siguiente: en el apartado dos se realiza una revisión de la literatura sobre competencia y poder de mercado, además de su impacto en las empresas: En el tercero se analiza la importancia de la microempresa en Baja California y el nivel de marginación socioeconómica de sus principales regiones; en el cuarto se desarrolla la metodología utilizada para contrastar la hipótesis de poder de mercado en microempresas. En el quinto se discuten los resultados obtenidos y finalmente se presentan las conclusiones.

\section{Competencia y poder de mercado en microempresas}

El análisis convencional de la microempresa parte de una función de producción neoclásica, en la cual se busca lograr una asignación óptima de recursos que permita obtener los beneficios más altos posibles. Para ello, el análisis del comportamiento de esas empresas se ha apoyado en los fundamentos del modelo de competencia perfecta, dando por hecho dos cuestiones: a) que éstas son agentes cuyo objetivo final es la maximización de beneficios y que, por tanto, en el corto plazo la cantidad de producción debe ser fijada en la intersección de las curvas de ingreso marginal y costo marginal, que ocurrirá en la parte ascendente de esta última; y $b$ ) que cualquier firma que opere en la industria carece de poder de mercado, debido a que compite en condiciones homogéneas y con información perfecta, por lo cual la curva de demanda que enfrenta de manera individual es horizontal. Ese modelo de competencia perfecta ha sido criticado por diversos autores debido a la rigidez de los supuestos en los cuales se basa. Robinson (1934:104), Schumpeter (1947:149),

Kirzner (1997:60) y Hayek (1945:519) abogan por un análisis con mayor 
fundamentación empírica que se podría enriquecer mediante la flexibilidad de supuestos y la consideración de otros factores.

Generalmente se acepta que la teoría neoclásica del equilibrio competitivo sea válida sólo si la función de demanda de cada firma en la industria es perfectamente elástica (Robinson, 1934:104). Para ello existen dos condiciones mínimas muy difíciles de cumplir: 1) que el número de firmas vendiendo un determinado producto sea suficientemente grande para que el efecto de un incremento en la producción individual o en el precio de mercado del bien sea insignificante; y 2) que el producto sea perfectamente homogéneo como para que los compradores no tengan razón alguna para preferir los bienes de una compañía sobre los de otra, excepto por las diferencias en precios (White, 1936:637). A su vez, ello requerirá que las empresas no tengan capacidad para diferenciar sus productos y que la tecnología sea un factor exógeno.

Para que la falta de capacidad de diferenciación sea posible, los gustos y preferencias de los demandantes por los bienes deben ser iguales, lo cual es muy difícil de darse en la realidad, pues un producto puede ser distinto ante los ojos de los consumidores por diferentes razones, como la facilidad para adquirirlo, costos de transacción (transporte), facilidades de compra, horario, tiempo de compra, facilidades de pago, presentación, entre otras (Cabral, 2000).

En el caso del supuesto de tecnología exógena, existen dos posturas. Por un lado, los autores de la teoría del crecimiento exógeno sostienen que los productores pueden acceder a la tecnología sin importar sus características individuales. Dada la no rivalidad y no exclusividad de la tecnología, las firmas se ven incapacitadas de ejercer poder de mercado respecto de sus adversarios, puesto que sus funciones de costos tienden a homogenizarse (Solow, 1956:65). Por otro lado, autores de la teoría del crecimiento endógeno argumentan que si bien el factor tecnológico puede ser exógeno, su papel de bien público no tiene cabida en ninguna estructura competitiva, menos en el corto plazo, cuando la exclusividad de algún invento o innovación es total. La invención de maquinaria para reducir costos, un aprovechamiento mayor de las habilidades empresariales, o bien, una nueva forma de combinar los factores de producción, se verán recompensados con la capacidad para limitar la competencia mediante costos minoritarios o vender por encima del precio de mercado (Romer, 1994:3-22). Según Schumpeter (1947:149), es precisamente ese poder de mercado que confiere beneficios de monopolio el que motiva la innovación y el descubrimiento. 
Finalmente, Palacio (2002) argumenta que cuando la tecnología es endógena se compite primordialmente por medio de la innovación. En tal caso, la diferenciación y diversificación del producto y la integración vertical son estrategias básicas de competencia en vez de barreras de entrada. El poder de mercado puede ser el reflejo de una mayor capacidad competitiva y no necesariamente de una situación de restricción a la competencia.

Dada la rigurosidad de los supuestos que rigen el modelo de competencia perfecta y sus objeciones a la aparición de poder de mercado, se empieza a revisar la posibilidad de que la microempresa encuentre una explicación más convincente si se analiza en otro marco de competencia. Una posibilidad está en relacionarla con el modelo de competencia monopolística, presumiendo que la diferenciación del producto mediante la localización geográfica, el desempeño de los trabajadores y las habilidades empresariales de los propietarios les puedan permitir actuar con poder de mercado a pesar de las restricciones tecnológicas con que operan.

White (1936:637) asume que un mercado monopolísticamente competitivo puede desplazar hacia afuera su curva de demanda por medio del incremento en la calidad, de la habilidad en el manejo de la mercadotecnia y del precio. Cuando los productores no pueden obtener mayores beneficios por medio de la manipulación del precio, la única alternativa es incrementar el grado de diferenciación del producto.

En su análisis del equilibrio de competencia monopolística, Chamberlin (1933) resalta cuatro conclusiones centrales: los precios son mayores que los de competencia perfecta por la diferenciación del producto; la escala de producción agregada puede ser mayor o menor que la escala óptima que pudiera ser alcanzada en competencia perfecta; las ganancias pueden ser mayores que aquellas en competencia perfecta y no necesariamente se deben a las imperfecciones del mercado; y existe la posibilidad de un flujo persistente de empresas hacia el mercado, aun cuando los beneficios en la industria no son excepcionales, debido quizás a un optimismo transitorio o mal cálculo de las ganancias que la industria está experimentando (White, 1936:637-649).

Por su parte, Demsetz (1959:21) propone que la principal característica que distingue esa estructura de mercado es la creación de un producto diferenciado en manos de un número considerable de firmas que se protege de la entrada de mayor número de competidores con los medios que las leyes de un gobierno confieren, entre ellas, los trademark, los derechos de autor y las patentes. Con esas barreras, el supuesto de entrada libre no tiene validez para explicar el tradicional equilibrio de beneficios cero en competencia monopolística de Chamberlin (Capozza y Robert, 1982:27). 
No obstante, existe una postura recientemente expuesta por Borch et al. (1999:145) con aplicación a la micro y pequeña industria de alimentos de los países nórdicos. El estudio propone cuatro estrategias de posicionamiento competitivo, que una vez combinadas ayudan a diferenciar a las empresas de acuerdo con sus capacidades directivas y productivas, generando poder de mercado y restringiendo, por tanto, la competencia. La primera es llevar a cabo una diferenciación del cliente mediante la adaptación del producto a las necesidades del mismo, tomando en cuenta la edad, educación, nivel de ingresos, estatus social, entre otros; la segunda se relaciona con la diferenciación del producto, mediante el sabor, color, consistencia, empaque y hasta la forma en que se produce la materia prima (Tregaer, 1998); la tercera trata del nivel del precios, enfatizando la necesidad de sobreponerse a la dificultad que enfrentan las empresas pequeñas en fijar sus precios debido al desconocimiento del mercado; y, por último, la cuarta, que se refiere al uso de los canales de distribución.

Por otra parte, Hall (1993:607) encuentra que el know-how del director o empresario es el recurso intangible más importante para que un negocio tenga éxito. Por tanto, uno podría esperar que quienes tienen una educación y experiencia amplias sean más creativos al momento de canalizar los recursos hacia nuevas formas de crecimiento. Asimismo, pudieran tener mayor habilidad para incorporar recursos externos y llevar a cabo innovaciones en la empresa, por ejemplo, el desarrollo de un nuevo producto, la inclusión de un nuevo mercado o la incorporación de una tecnología más avanzada (Borch, et al., 1999:1-45).

\section{Microempresa y marginación socioeconómica en Baja California}

El estado de Baja California se caracteriza por una actividad económica pujante, que en parte se asocia a su cercanía con California, una de las economías más dinámicas de Estados Unidos y del mundo. Ello le ha permitido tener un ritmo de crecimiento considerable en los últimos años, liderado sobre todo por la industria maquiladora de exportación.

La economía de Baja California se ha distinguido por una participación muy importante de la industria manufacturera, especialmente la maquiladora. Su contribución al PIB del estado en 1999 fue de $22.3 \%$, desplazando inclusive a las actividades de comercio y hotelería que tradicionalmente habían sido las que mayor participación reportaban (Ocegueda, 2003:1024). Sin embargo, no es el sector que mayor 
Cuadro 1

Número de establecimientos por municipios en Baja California, 2002

\begin{tabular}{lccccc}
\hline $\begin{array}{c}\text { Municipio } \\
\text { Empresas }\end{array}$ & Microempresa & Pequeña & Mediana & Grande & Total \\
\hline Ensenada & 1935 & 212 & 102 & 28 & 2277 \\
& $17.9 \%$ & $18.9 \%$ & $16.5 \%$ & $8.0 \%$ & $17.6 \%$ \\
Mexicali & 3540 & 314 & 132 & 91 & 4077 \\
& $32.7 \%$ & $27.9 \%$ & $21.3 \%$ & $25.9 \%$ & $31.6 \%$ \\
Tecate & 274 & 32 & 20 & 5 & 331 \\
& $2.5 \%$ & $2.8 \%$ & $3.2 \%$ & $1.4 \%$ & $2.6 \%$ \\
Tijuana & 4921 & 562 & 361 & 227 & 6071 \\
& $45.5 \%$ & $50.0 \%$ & $58.3 \%$ & $64.7 \%$ & $47.0 \%$ \\
Playas de Rosarito & 141 & 4 & 4 & 0 & 149 \\
& $1.3 \%$ & $0.4 \%$ & $0.6 \%$ & $0.0 \%$ & $1.2 \%$ \\
Total & 10811 & 1124 & 619 & 351 & 12905 \\
Participación & $83.8 \%$ & $8.7 \%$ & $4.8 \%$ & $2.7 \%$ & $100 \%$ \\
\hline
\end{tabular}

Fuente: Sistema de Información Empresarial Mexicano, 2002.

número de empresas reporta en el estado. Ese lugar sigue siendo ostentado por el sector comercio, pues de acuerdo con el Sistema de Información Empresarial Mexicano (SIEM), en 2002, del total de establecimientos en la entidad, 53.4\% se concentraban en ese sector, seguido por el de servicios con $24.3 \%$, perteneciendo el resto al sector industrial (22.3\%).

No obstante, el dominio en la estructura empresarial no le pertenece a la gran empresa, sino a una fuerte concentración de microempresas que se ubican en las diferentes ramas de la actividad económica. Los datos del Cuadro 1 indican que del total de empresas, $83.8 \%$ corresponde a la clasificación de microempresas que se distribuyen en los diferentes sectores de la economía, siendo el sector comercio donde más proliferan. La estructura empresarial del estado se reproduce de manera muy similar en los diferentes municipios. En el caso de Tijuana, ese comportamiento se explica, en parte, por su cercanía con el condado de San Diego, que ha provocado una vigorosa dinámica empresarial, y a la incesante llegada de residentes de otras partes de la república en busca de oportunidades, y en el caso de Mexicali, obedece, en buena medida, al hecho de ser la capital de la entidad y a su desarrollo agroindustrial. Cuarenta y siete por ciento del total de las empresas del estado se concentra en el municipio de Tijuana y $31.6 \%$ en Mexicali.

El municipio de Ensenada participa con $17.6 \%$ de las empresas establecidas en la entidad y un porcentaje muy elevado de ellas son básicamente microempresas 
(85\%) que desarrollan diversas actividades, entre las que destacan el comercio y el turismo. El reducido número de grandes empresas ubicadas en este municipio se dedica a industrializar productos marítimos y agroindustriales, si bien algunas son empresas maquiladoras que aprovechan las ventajas de ese puerto e introducen sus productos a California por ese medio. El resto de los municipios (Tecate y Playas de Rosarito) tienen una representación mínima en la estructura empresarial del estado, pues apenas logran una participación de 2.6 y $1.2 \%$, respectivamente, con una inmensa mayoría de micro y pequeñas empresas. La estructura empresarial de los diversos municipios del estado revela la importancia del sector microempresarial y la necesidad de favorecer su desarrollo para hacerlas más eficientes y consolidarlas como opción de vida para quienes viven de ellas.

A pesar de que Baja California es uno de los estados con mayor dinamismo industrial y menor índice de marginación socioeconómica en el nivel nacional (CONAPO, 2001) la pobreza está presente en un gran porcentaje de familias migrantes en colonias irregulares, cuyos ingresos para sostener al hogar dependen de una pequeña actividad económica como la microempresa, y donde los esfuerzos del propietario y de su familia son insuficientes para generar el capital de reinversión necesario que promueva su crecimiento. En consecuencia, muchos de esos micronegocios se encuentran cautivos en una trampa de subsistencia (Mungaray et al., 2005:316).

Hablar de marginación en ese contexto se relaciona con un tipo de exclusión socioeconómica que impide el goce de beneficios tecnológicos, económicos y de infraestructura entre grupos sociales de productores y consumidores, a quienes la falta de educación, ingreso o información les impide la satisfacción óptima de sus necesidades básicas. La marginación se origina en el patrón de desarrollo de una región y tiene dos manifestaciones principales: la primera es la dificultad para propagar el progreso técnico en el conjunto de la estructura productiva y en las regiones, y la segunda es una exclusión de individuos del proceso de desarrollo y del disfrute de sus beneficios (CONAPO, 2001). Esa marginación puede referirse a una carencia económica en la producción o el consumo cultural, educacional, étnico y de valores de ciertos grupos respecto de otros, y propone causas de orden económico-social, político-social, educacional, cultural, psicosocial y demográfico (CONEPO, 1995).

Si bien es cierto que la marginación en las colonias desfavorece el intercambio de bienes y servicios, debido a que es mucho más costoso para los productores y los 
consumidores, ésta ha permitido que por lo menos en una concentración territorial, microempresas con escasa tecnología, capacidad instalada limitada y nulo apoyo financiero, puedan encontrar dinámicas de subsistencia por medio de la fijación de precios por encima de sus costos marginales.

Desde el punto de vista de la producción, la marginación implica barreras a la entrada para competidores de magnitud superior a las microempresas. Una de esas barreras se da cuando el pequeño, mediano o gran empresario prevé beneficios cero o negativos derivados de emprender un negocio en comunidades de bajos ingresos y con baja elasticidad en la demanda a los cambios de precios, derivado de la insolvencia económica. Por otro lado, la desmotivación también puede surgir ante la amenaza que representa llevar a cabo actividades empresariales en regiones con altos niveles de inseguridad pública imperantes, dada la carencia de vigilancia y vialidades adecuadas, además de los costos de transporte, inversión de tiempo y riesgos en materia de seguridad que implican llevar el bien a localidades con condiciones orográficas sumamente desfavorables.

Desde el punto de vista del consumidor, la marginación trae consigo costos que benefician directamente al productor ubicado en esas zonas geográficas. Debido a la lejanía de las localidades marginadas y a los costos de transporte, de tiempo y de oportunidad que implica acceder a los comercios céntricos, los propietarios de micronegocios tienen la justificación para fijar precios por encima de aquellos bienes de zonas no marginadas y los consumidores están dispuestos a pagarlos. Entonces, la propia situación de marginación y la necesidad del cliente son causas que le generan poder de mercado al microproductor y le permite sobrevivir, al menos en el corto plazo, en el entendido de que si éste no tiene capacidad para reinvertir, reducir costos o innovar a través del aprendizaje empresarial, difícilmente logrará sobrevivir en el largo plazo.

\section{Metodología y fuentes de información}

\section{El modelo econométrico}

Para corroborar la hipótesis de poder de mercado en microempresas, es necesario construir un indicador que lo mida en cada una de las mismas. Una manera de hacerlo es mediante el índice diseñado por Lerner (1934:157-175), el cual es concebido como un indicador confiable en el corto plazo (Clarke, 2000). Este índice se puede estimar para las empresas mediante la siguiente ecuación: 


$$
L_{i t}=\left(\frac{p_{i t}-C m g_{i t}}{p_{i t}}\right)
$$

en la cual, para el momento $t, L_{i t}$ equivale al índice de Lerner de la microempresa $i ; p_{i t}$ representa el precio del producto de la microempresa $i$, y $\mathrm{Cmg}_{i t}$ es el costo marginal en que incurre la microempresa $i$. Si bien es cierto que Lerner propone tomar en cuenta el costo marginal como una medida de desembolso económico empresarial, Huergo (2001) menciona que debido a la inobservabilidad de éste y en el supuesto de rendimientos constantes a escala, el índice de Lerner puede ser construido con base en los costos medios $(\mathrm{Cme})$. De esa forma, la medición se vuelve empíricamente más conveniente para no poner en riesgo la confiabilidad del análisis. Por tanto, el índice de Lerner en este estudio queda representado por la siguiente ecuación:

$$
L_{i t}=\left(\frac{p_{i t}-C m e_{i t}}{p_{i t}}\right)
$$

en la cual, $C m e_{i t}$ representa el costo medio de la microempresa $i$.

Se construye el modelo econométrico con el objetivo de mostrar evidencia empírica en favor del poder de mercado en microempresas (Aguilar y Barrón, 2006) y argumentar qué tanto puede ser explicado por factores exógenos, como la diferenciación de sus productos que logran de manera natural al concentrarse en mercados marginados, y por factores endógenos como la productividad de sus trabajadores, la capacidad del empresario para innovar y la capacidad productiva de la microempresa, entre los más importantes. Dicho modelo mide la relación que existe entre el índice de Lerner del grupo de microempresas que se considera en la muestra y dos vectores de variables explicativas; el primero agrupa variables de carácter endógeno, es decir, que dependen del comportamiento propio de la empresa, mientras que el segundo contempla variables exógenas a la empresa, como es el índice de marginación de la colonia donde está ubicada la misma. El modelo se representa mediante la siguiente ecuación:

$$
L_{i t}=\alpha-\beta_{1} X_{i t}-\beta_{2} Y_{i t}-u_{i t}
$$


en la cual, $L_{i t}$ es el índice de Lerner de la microempresa $i$. Además, $\alpha, \beta_{1}$ y $\beta_{2}$ son parámetros que representan el intercepto de la función, el primero, y el segundo y tercero miden la pendiente y la relación entre $L_{i t}$ y dos vectores de variables $X_{i t}$ y $Y_{i t}$. El primero de esos vectores representa las variables endógenas que pueden incidir en la capacidad de la empresa para adquirir poder de mercado, el cual incluye el índice de productividad de los trabajadores, la educación del dueño y la capacidad productiva de la empresa, representada por la diferencia entre la producción óptima que permite minimizar los costos medios de la empresa en el corto plazo y la producción promedio por jornada laboral.

El índice de productividad de los trabajadores se estimó mediante el siguiente procedimiento:

$$
I p_{i t}=\frac{V A_{i t}}{n_{i t}}
$$

en el cual, para el momento $t, I p_{i t}$ es el índice de productividad de los trabajadores de la empresa $i ; V A_{i t}$ mide el valor agregado total de la empresa; $i$ y $n_{i t}$ constituye el número total de trabajadores de la empresa $i$.

La inclusión en el modelo de variables explicativas como el índice de productividad de los trabajadores o la educación del dueño, tienen la intención de medir el desempeño del factor trabajo y las habilidades empresariales en la mejora de la eficiencia de la empresa, que se manifiesta mediante reducción de costos y mejoras en los procesos de innovación, planeación, organización y estrategias de ventas, entre otras. Todas esas acciones favorecen la aparición de poder de mercado que la empresa posee. A su vez, la variable identificada como capacidad productiva de la compañía tiene la intención de medir cómo el hecho de producir por debajo o por encima del nivel óptimo que minimiza los costos medios en el corto plazo (y que garantiza la permanencia de la microempresa en el mercado), impacta en el incremento o reducción de los costos derivados del nivel de producción que maneja la empresa. Por tanto, se esperaría una relación positiva entre el índice de Lerner y esa variable.

En el caso del segundo vector de variables, dada la complejidad que conlleva capturar efectos externos a las empresas, tales como gustos y preferencias de los consumidores o costos de transacción, $Y_{i t}$ sólo representa el índice de marginación por colonia, en el supuesto de que esas empresas logran diferenciar sus productos 
por el simple hecho de ubicarse en distintas colonias o mercados marginados. Ese índice capta el grado de marginación socioeconómica asociado con cada una de las colonias a las que pertenecen las microempresas de la muestra y que constituye un aspecto fundamental en este trabajo, pues de comprobarse una relación entre éste y el indicador de poder de mercado, se afirmaría que las carencias socioeconómicas en cierta áreas territoriales dan pie a que se creen situaciones de mercado en las cuales los micronegocios operantes gozan de privilegios que no son concebibles en una estructura estrictamente competitiva.

El índice de marginación por colonia fue construido con base en la metodología utilizada por el CONAPO para calcular el índice de marginación estatal y municipal en México para 2000 (Aguilar y Barrón, 2006) y en la cual se utiliza la técnica de componentes principales. Ésta se logra estimando la siguiente ecuación:

$$
I M_{j}=\sum_{i=1}^{w} F P_{i} \frac{X_{i j}-\bar{X}_{i}}{d_{i}} \quad j=1,2 \ldots, N
$$

en la cual, $I M_{j}$ es el índice de marginación de la unidad geográfica $j ; X_{y}$ corresponde al valor del indicador $i$, de la unidad geográfica $j ; \overline{X_{i}}$ representa el valor de la media del indicador $i ; d_{i}$ denota la desviación estándar del indicador $i ; F P_{i}$ es el factor de ponderación del indicador $i ; N$ es el número de la muestra considerada en el estudio; y $w$ es el número de indicadores de marginación utilizados. Los indicadores denotados por la variable $i$ corresponden a aspectos sobre el nivel de marginación de la población como población analfabeta, viviendas sin drenaje, niveles de ingreso, entre otros.

Para estimar la ecuación 5 se hace uso de los datos proporcionados por el Sistema de Consulta para la Información Censal por Colonias (SCINCE) de 2000. La estimación del índice de marginación no fue realizada con todos los indicadores utilizados por la metodología del CONAPO, debido a la falta de disponibilidad de datos que implica la elaboración a nivel "colonia" del índice. Sin embargo, se definieron cuidadosamente otros indicadores que tuvieran validez similar que los recomendados, quedando las siguientes variables como las más importantes para tal efecto: 1) porcentaje de la población analfabeta de 15 años o más; 2) porcentaje de la población de 15 años y más con primaria incompleta; 3) grado de escolaridad promedio de la población de 15 años y más; 4) porcentaje de viviendas particulares 
habitadas sin drenaje; 5) porcentaje de viviendas particulares habitadas con techos de materiales ligeros, naturales o precarios; 6 ) porcentaje de viviendas particulares habitadas sin disponibilidad de energía eléctrica; 7) porcentaje de viviendas particulares sin disponibilidad de agua entubada en la vivienda; 8) promedio de ocupantes por cuarto en viviendas particulares habitadas; 9) porcentaje de viviendas particulares sin pisos de cemento, mosaico, madera u otro recubrimiento; 10) porcentaje de la población ocupada sin ingresos o hasta dos salarios mínimos.

Una vez construido el índice de marginación, éste se clasificó en cinco categorías, con el fin de diferenciar entre las colonias con mayor y menor marginación (CONEPO, 1995). Para categorizar, se considera con marginación muy baja aquellas que su índice sea menor a la media menos 1.5 de la desviación estándar; baja si el índice se encuentra entre la media menos 0.5 y la media menos 1.5 veces la desviación estándar; media si está entre la media más/menos 0.5 la desviación estándar; alta si se localiza entre la media más 0.5 y la media más 1.5 veces la desviación estándar; y muy alta si es índice superior a la media más 1.5 la desviación estándar.

Una vez estimadas las variables de cada uno de los vectores de variables, se aplicó logaritmo en aquellas donde fue posible con el fin de minimizar la varianza y normalizar las series. El modelo a estimar se representa mediante la siguiente ecuación:

$$
l_{i t}=\alpha-\beta_{1} I m_{i t}-\beta_{2} I p_{i t} \beta_{3} C p_{i t}-\alpha_{e} D e_{i t}-\alpha_{c} D c_{i t}-\alpha_{m} D m_{i t}-u_{i t}
$$

en la cual $l_{i t}$ representa el logaritmo natural del índice de Lerner de la microempresa $i ; I_{i t}$ es el logaritmo del índice de marginación de la colonia donde está establecida la microempresa $i ; I p_{i t}$ es logaritmo del índice de productividad de los trabajadores de la microempresa $i$, y $C p_{i t}$ es la capacidad productiva de la microempresa $i$. Los coeficientes $\alpha, \beta_{1} \beta_{2}$ y $\beta_{3}$ son los parámetros por estimar. Adicionalmente y con el fin de medir el impacto que puede tener la educación de los dueños de las empresas, se incorporó la variable dicotómica $D e_{i t}$ que toma el valor de 1 si el propietario tiene educación primaria o secundaria y 0 en caso de tener educación inferior. Por otra parte, para controlar los efectos derivados del nivel de infraestructura y desarrollo de la ciudad donde está ubicada la microempresa y el nivel de marginación de la colonia donde se encuentra, se incorporaron las variables dummy $D c_{i t}$ y $D m_{i t}$. Dado que la mayoría de las empresas de la muestra se localizan en Tijuana, $D c_{i t}$ toma el valor de 1 si la microempresa $i$ se ubica en esta ciudad y 0 en caso de ubicarse en 
Mexicali; y la variable $D m_{i t}$ toma el valor de 1 si el nivel de marginación de la microempresa $i$ es alto o muy alto y 0 en caso de ser medio, bajo o muy bajo. Por último, el término $u_{i t}$ representa el error estocástico de la función. Los parámetros $\alpha_{e}, \alpha_{c}$ y $\alpha_{m}$ capturan el efecto que pueden tener en el intercepto de la función, el nivel de educación de los dueños, la ubicación y el nivel de marginación de las microempresas.

Datos y fuentes de información

Para estimar la ecuación 6 se utilizó información de 29 microempresas ubicadas en zonas marginadas de Tijuana y Mexicali, cuyo giro es la elaboración y comercialización de productos alimenticios, entre los cuales destacan: tamales, burritos, panes, pasteles, entre otros. Recopilar esta información se logró gracias al Programa de Investigación, Asistencia y Docencia a la Micro y Pequeña Empresa que desde 2001 se ha venido aplicando en la Facultad de Economía de la Universidad Autónoma de Baja California, el cual consiste en brindar asistencia técnica en materia de administración, producción, costos y rentabilidad a microempresas marginadas ubicadas en las diferentes localidades del estado, mediante prestadores de servicio social.

El tamaño de la muestra se determinó con un procedimiento no probabilístico en virtud de la informalidad, marginación e inestabilidad de esos comercios y sus actividades productivas, que hace imposible conocer con precisión su número exacto y ubicación geográfica. En cualquier caso, la determinación de la muestra y el análisis de la misma ignora a las empresas formales de cualquier tamaño y la interacción de éstas con las informales.

El Cuadro 2 muestra que las empresas de la muestra utilizan en promedio dos trabajadores, incluyendo al dueño, y cuentan con un valor promedio en sus activos fijos que no rebasa los 12,500 pesos, lo cual indica que esas empresas son artesanales y que muchas de ellas producen en el seno de sus hogares y con tecnologías tradicionales. Las ventas promedio semanales no superan los 4,100 pesos, aunque es importante mencionar que existe una desviación estándar significante, pues las microempresas que se dedican a la elaboración de pan logran tener ventas muy superiores a las del resto de la muestra. Un dato importante es el de los beneficios promedio que generan, pues sólo alcanzan la cifra semanal de 2,266 pesos, lo cual deja claro la poca capacidad de estas empresas para acumular capital. 
Cuadro 2

Características de microempresas por tipo de actividad

\begin{tabular}{|c|c|c|c|c|c|c|}
\hline \multirow[b]{2}{*}{ Giro } & \multirow[b]{2}{*}{ Empresas } & \multirow{2}{*}{$\begin{array}{l}\text { Valor de } \\
\text { activos en pesos } \\
\text { (promedio) }\end{array}$} & \multirow{2}{*}{$\begin{array}{c}\text { Trabajadores } \\
\text { (promedio) }\end{array}$} & \multicolumn{3}{|c|}{ Pesos semanales (promedio) } \\
\hline & & & & Ventas & Costos & Beneficios \\
\hline Tortillería & 4 & 14691.00 & 2.0 & 2539.85 & 1556.18 & 977.34 \\
\hline Panadería* & 5 & 25359.75 & 2.0 & 8395.43 & 2787.20 & 5683.12 \\
\hline Tamales & 13 & 3123.89 & 1.6 & 1761.98 & 965.59 & 787.43 \\
\hline Otros & 7 & 6767.80 & 1.8 & 3417.16 & 1771.72 & 1616.63 \\
\hline Total & 29 & 12485.61 & 1.85 & 4028.60 & 1770.17 & 2266.13 \\
\hline
\end{tabular}

* Incluye producción de pasteles

Fuente: elaboración propia con datos del PIADMyPE.

\section{Resultados}

El Cuadro 3 muestra que de las 29 microempresas analizadas, 69\% se encuentra ubicado en colonias que corresponden a una clasificación de marginación alta y muy alta. De acuerdo con este cuadro, la media del índice de Lerner en la muestra fue de 0.45 con $38 \%$ de las observaciones mostrando un valor por encima de este valor medio, lo que da una primera evidencia estadística en favor de la hipótesis planteada en este trabajo, el cual sostiene que microempresas con características de marginación son capaces de generar cierto poder monopolístico en sus nichos de mercado. Ello indicaría que ésta no es una cualidad exclusiva de las grandes empresas, sino que también puede ser encontrada en mercados pequeños donde la dimensión, localización e infraestructura y otras manifestaciones de la marginación le podrían estar confiriendo cierto poder de mercado. Otro resultado importante es que la correlación entre el índice de Lerner y el grado de marginación es más fuerte a medida que el nivel de marginación crece.

Los resultados econométricos se muestran en el Cuadro 4, en el cual se puede apreciar que son significativos estadísticamente, pues la prueba de White señala que el problema de heteroscedasticidad, que puede ser normal en ejercicios de corte transversal, está ausente. Ello permite rechazar la hipótesis de resultados espurios. De igual forma, la prueba de Ramsey permite deducir que existe una correcta especificación del modelo, a pesar de no tratarse de un modelo totalmente teórico, sino deducido empíricamente del comportamiento de estas empresas. En todo caso, la teoría neoclásica afirmaría que estas empresas por su tamaño, capacidad productiva y nivel tecnológico son incapaces de poseer poder de mercado. 
Cuadro 3

Correlación entre el índice de Lerner y el de marginación

\begin{tabular}{|c|c|c|c|c|c|c|c|}
\hline Microempresa & Colonia & Ciudad & Giro & ILer & IMarg & NMarg $I$ & ICorr \\
\hline Delfina Cruz Rivera & Cachanilla & Mexicali & Tortilla de harina & 0.39 & 0.89 & Muy alta & a 1 \\
\hline Catalina Castro & Ampliación Solidaridad & Mexicali & Tortilla de harina & 0.13 & 0.56 & Media & 0.28 \\
\hline Andrea Nevárez & Solidaridad & Mexicali & Nopales & 0.57 & 0.56 & Media & 0.28 \\
\hline Fausto Arellanes & Las Torres, Otay & Tijuana & Panadería & 0.70 & 0.64 & Alta & 0.34 \\
\hline Gamaliel Patiño & Emiliano Zapata & Tijuana & Tamales & 0.32 & 0.79 & Alta & 0.34 \\
\hline Guadallupe Valdez Osorio & Rinconada, Otay & Tijuana & Tamales & 0.48 & 0.68 & Alta & 0.32 \\
\hline María Lourdes Cossio & Playas de Tijuana & Tijuana & Pastelería & 0.33 & 0.21 & Baja & 0.32 \\
\hline Hernández Delia & Las Torres, Otay & Tijuana & Pastelería & 0.74 & 0.64 & Alta & 0.34 \\
\hline María Onésima González & Mariano Matamoros & Tijuana & Tamales & 0.44 & 0.65 & Alta & 0.34 \\
\hline Agustina Ruiz Contreras & Las Torres, Otay & Tijuana & Tamales & 0.38 & 0.64 & Alta & 0.34 \\
\hline María G. Salazar Bueno & Rinconada I, Otay & Tijuana & Tamales & 0.24 & 0.68 & Alta & 0.32 \\
\hline María Guadalupe Saucedo & Mariano Matamoros & Tijuana & Tortillería & 0.46 & 0.65 & Alta & 0.34 \\
\hline Patricio Soto & Mariano Matamoros & Tijuana & Torilla de harina & 0.40 & 0.65 & Alta & 0.34 \\
\hline Guadalupe V. Osorio (2) & Rinconada Otay & Tijuana & Tamales & 0.55 & 0.68 & Alta & 0.32 \\
\hline María Onésima & Mariano Matamoros & Tijuana & Tamales & 0.54 & 0.65 & Alta & 0.34 \\
\hline \multicolumn{8}{|l|}{ González (2) } \\
\hline Eufracia Gordián Rueda & 18 de Marzo & Tijuana & Tamales & 0.65 & 0.32 & Media & 0.28 \\
\hline Martha Leticia Haro & Ampliación Guaycura & Tijuana & Tamales & 0.57 & 0.40 & Media & 0.28 \\
\hline Orfa Vázquez Ruiz & Río parte baja & Tijuana & Burritos & 0.63 & 0.44 & Media & 0.28 \\
\hline Prisciliana Lopez Inojo & Cañón Sainz & Tijuana & Tamales & 0.61 & 1.00 & Muy Alta & a 1 \\
\hline Guadalupe Mendoza & Terrazas del Valle & Tijuana & Helados & 0.48 & 0.43 & Media & 0.28 \\
\hline \multicolumn{8}{|l|}{ Méndez } \\
\hline Camerina Preciado & Independencia & Tijuana & Tamales & 0.29 & 0.31 & Media & 0.28 \\
\hline \multicolumn{8}{|l|}{ Carmona } \\
\hline Gloria Leticia Santillanes & Lomas del Matamoros & Tijuana & Tamales & 0.45 & 0.49 & Media & 0.28 \\
\hline Jesús Serrano & 18 de Marzo & Tijuana & Tamarindo & 0.52 & 0.32 & Media & 0.28 \\
\hline Guadalupe Valdez & Rinconada Otay & Tijuana & Tamales & 0.53 & 0.68 & Alta & 0.32 \\
\hline \multicolumn{8}{|l|}{ Osorio (2) } \\
\hline María Rodríguez & Rinconada I, Otay & Tijuana & Flanes & 0.42 & 0.68 & Alta & 0.32 \\
\hline \multicolumn{8}{|l|}{ Hernández } \\
\hline Patricio Soto (2) & Mariano Matamoros & Tijuana & Burritos & 0.25 & 0.65 & Alta & 0.34 \\
\hline Francisco Pedraza & Ampliación Sánchez & Tijuana & Tacos & 0.28 & 0.65 & Alta & 0.34 \\
\hline & Tabeada & & & & & & \\
\hline Ricardo Vilchis Rico & Rinconada I, Otay & Tijuana & Panes & 0.17 & 0.68 & Alta & 0.32 \\
\hline Salvador B. Manzanares & Mariano Matamoros & Tijuana & Panes & 0.55 & 0.65 & Alta & 0.34 \\
\hline Media & & & & 0.45 & 0.45 & & 0.36 \\
\hline Desviación estándar & & & & 0.16 & 0.29 & & 0.17 \\
\hline
\end{tabular}

Fuente: elaboración propia con datos del PIADMyPE.

ILer = índice de Lerner; IMarg = índice de marginación; NMarg = nivel de marginación; ICorr = índice de correlación. (2) = empresas atendidas en etapas diferentes. 
Cuadro 4

Resultados econométricos

\begin{tabular}{lcccccccccccc}
\hline & $\alpha$ & $\beta_{1}$ & $\beta_{2}$ & $\beta_{3}$ & $\alpha_{e}$ & $\alpha_{c}$ & $\alpha_{m}$ & $R^{2}$ & $D W$ & $F$ & $\begin{array}{c}\text { Prueba Prueba } \\
\text { White Ramsey }\end{array}$ \\
\hline Coeficiente & - & 0.7 & & 0.00 & & & & 0.2 & 1.7 & & 0.4 & 1.0 \\
& & & 0.15 & & 0.14 & 0.57 & 0.50 & & & 2.25 & & \\
& 0.79 & 3 & & 1 & & & & 1 & 7 & & 8 & 2 \\
(testadístico) & $(-3.45)$ & $(1.95)$ & $(2.53)$ & $(1.69)$ & $(0.80)$ & $(1.96)$ & $(1.96)$ & & & & & \\
{$[$ [Probabilidad] } & {$[0.002]$} & {$[0.06]$} & {$[0.01]$} & {$[0.10]$} & {$[0.42]$} & {$[0.06]$} & {$[0.06]$} & & & {$[0.06]$} & {$[0.89]$} & {$[0.44]$} \\
\hline
\end{tabular}

Fuente: elaboración propia con datos del PIADMyPE.

El hecho de que se presente un $\mathrm{R}^{2}$ (ajustado) bajo, no es suficiente para invalidar los resultados, puesto que las observaciones utilizadas corresponden a un análisis de corte transversal, razón por la cual no es indispensable esperar que este indicador sea elevado. Además, el valor de $t$ de los coeficientes estimados, a excepción de la variable dummy, $D e_{i t}$, son significativos en un nivel de confianza de 90 y $95 \%$, y el $F$ estadístico que muestra la significancia conjunta del modelo es significativo a $94 \%$ de confianza.

Los resultados del modelo muestran la existencia de un grado de asociación muy considerable entre las variables explicatorias y el índice de Lerner, además de que los signos de los coeficientes son los esperados. La alta significancia de los parámetros $\beta_{1}, \beta_{2}$ y $\beta_{3}$ muestra que variables como el nivel de marginación, productividad de los trabajadores y capacidad productiva de la empresa inciden en el poder de mercado que puede poseer una microempresa y en el hecho de que pueda fijar precios por encima del $\mathrm{Cmg}$ y $\mathrm{Cme}$. Entre ellas resalta el valor del $\beta_{1}(0.73)$, el cual es muy superior al resto, lo que indica que la capacidad de la empresa para actuar con poder de mercado depende mucho del nivel de marginación donde ésta se ubique. Este resultado se confirma con el valor del parámetro $\alpha_{m}$, pues su significancia demuestra un impacto positivo en el intercepto de la función, es decir, que las empresas que se encuentran en zonas de mayor nivel de marginación tienen mayor posibilidad para actuar monopolísticamente.

El hecho de que el coeficiente de la variable $D c_{i t}$ sea significativo, señala que el comportamiento de las empresas es diferente dependiendo de la colonia donde se ubique, pues a pesar de que Tijuana es una de las ciudades más industriales del estado, en ella existen zonas de alto nivel de marginación, debido en gran parte al crecimiento elevado de la inmigración, a la concomitante imposibilidad de crear la 
infraestructura adecuada y a la falta de articulación de las empresas locales con las grandes empresas. Todo ello ha inhibido que el beneficio de la industrialización llegue de manera inmediata hacia los sectores menos favorecidos en constante creación.

Por otra parte, si bien es cierto que el coeficiente de la variable $D e_{i t}$ no es significativa, la significancia conjunta del modelo permite deducir que la educación en estas empresas es fundamental para que se puedan realizar innovaciones y diseñar mejores mecanismos que permitan el desarrollo y competitividad de las mismas. Ello les permitiría insertarse en otros mercados de mayor capacidad de demanda, fuera de la protección que les puede brindar la marginación.

En esas condiciones, el modelo muestra que existe evidencia para corroborar la hipótesis de que microempresas marginadas de Baja California tienen poder de mercado, el cual está determinado principalmente por la marginación socioeconómica de la zona geográfica que habitan, por las habilidades empresariales de sus líderes y por el desempeño de sus trabajadores.

\section{Conclusiones}

El poder de mercado tiene costos considerables para la sociedad. En un esquema de producción con tendencias monopolísticas, es de esperarse que los precios sean mayores y las cantidades ofrecidas menores que en una situación de competencia, por lo cual el excedente de los consumidores tiende a reducirse a costa de un aumento en el de las empresas. En términos generales, hay una pérdida neta de bienestar para la sociedad, dada por una reducción en las compras, fruto de precios imperantes mayores y de un acotamiento de la producción debido a la maximización de beneficios empresariales a menores niveles productivos (Clarke, 2000).

No obstante, en microempresas marginadas como las de la muestra estudiada, el poder de mercado representa un medio de supervivencia dado el contexto en el que compiten. La marginación socioeconómica en la que se desenvuelven representa todo un dilema. Por un lado, por medio del peculiar poder de monopolio que genera, garantiza la continuidad del negocio y, por el otro, es un factor de estancamiento e ineficiencia económica para el mismo.

Es cierto que la marginación socioeconómica representa una barrera para el posible ingreso de empresas competidoras, pero también lo es que constituya una barrera para que las empresas salgan a contender fuera de su área, ya que las condiciones competitivas en mercados no marginados son muy distintas. Por lo general, las microempresas pobres no tienen la capacidad para enfrentar, fuera de su región, 
a sus homónimos de colonias más privilegiadas en términos económicos. En otras palabras, al esfumarse la marginación desaparecen con ella los privilegios que las protegen, por lo cual las firmas de colonias de bajos recursos tenderían a quedar excluidas del mercado en la medida que en éste mejore la distribución del ingreso.

Ante esta situación, sería deseable desarrollar un esquema de promoción encaminado a crear empresas competitivas y eficientes: con potencial de crecimiento y con miras a incrementar el desarrollo social. Para ello resultaría conveniente considerar algunas de las ventajas con las que actualmente cuentan los micronegocios, entre ellas, seguir aprovechando e impulsando la productividad de los trabajadores y las habilidades empresariales, factores condicionantes del poder de mercado según los resultados del presente estudio. Igualmente es aconsejable prestar atención a las recomendaciones que hace Borch et al. (1999:1-45) para crear ventajas de posicionamiento competitivo, que si bien pudieran generar poder de mercado en el corto plazo, a la larga pueden ser el motor de crecimiento económico una vez que se lleven a cabo los procesos naturales de imitación. Dichas acciones deberán ser generalizadas y abarcar el mayor número de microempresas posibles, de manera que la competencia tienda a lo más perfecto posible para que de las innovaciones constantes y de la lucha por la reducción de los costos de producción también los consumidores resulten beneficiados y se puedan desvanecer las condiciones impuestas por la marginación.

En busca del objetivo anterior, el gobierno deberá jugar su parte en materia de desarrollo comunitario para impulsar el bienestar de esas regiones y de sus agentes económicos. Además, deberá contar con programas de apoyo financiero más flexibles para empresas de este tipo, destacando la idea del PIADMyPE, de crear un fondo de financiamiento para empresas pobres en el cual las firmas no arriesguen su patrimonio en caso de que las condiciones del entorno no las favorezcan con un préstamo para la inversión.

Para implantar todas esas acciones se requiere de una retroalimentación del gobierno y de las instituciones de educación superior para crear planes estratégicos de ayuda a dicho sector. Asimismo, es de suma importancia el enlace entre las universidades y el sector microempresarial para llevar a cabo el intercambio de información y la transferencia de tecnología. Al respecto, es útil el programa de servicio social establecido en esas instituciones, pues permite aprovechar la disposición y el conocimiento del estudiantado para brindar asesoría técnica, productiva, financiera y económica a empresas desorganizadas y sin planificación, con el único objetivo 
de mejorar su eficiencia en todos los sentidos. Acciones de esta naturaleza permitirían a las microempresas marginadas tener posibilidades de crecer y sobreponerse a los retos que la dinámica del mercado actual representa. Tales acciones también harán posible contrarrestar los efectos que el poder de mercado tiene sobre el bienestar social.

Es cierto que el resultado empírico de que estas empresas cobren un precio mayor al de competencia perfecta, y que este mark up esté relacionado positivamente con el grado de marginación del entorno, puede no deberse tan sólo al posible ejercicio de poder de mercado de las mismas. Entre otros, factores como la tasa de eficiencia en el uso de los recursos o el papel de las empresas grandes compitiendo en esos mercados podrían sugerir, en equilibrio, precios mayores a los de competencia perfecta. En el primer caso, sería la ineficiencia en el uso de los recursos lo que obligaría a esas firmas a fijar un precio por encima del competitivo, no obstante que la literatura reciente muestra que esas compañías son generalmente eficientes en el uso de los recursos. Por otro lado, aunque es cierto que las grandes empresas no están preocupadas por lo que en lo particular pueda hacer o dejar de hacer cada una de las microempresas, y que ello haría pensar que la competencia se relaja en esos entornos (y, por lo tanto, los precios irían a la alza), no se puede olvidar que en el mercado de la microempresa marginada la gran empresa sí juega un papel relevante (cuyo efecto no ha sido estudiado en este trabajo), estando cotidianamente presentes en radio, televisión y prensa y siendo siempre una opción para el consumidor, por lo cual no se puede descartar el interés de los propietarios de ese tipo de negocios por defender una posición competitiva en su nicho de mercado.

Bibliografía

Aguilar, José y Karla Barrón, “Poder de mercado y marginación de las microempresas: estudio de caso de Baja California", en Comercio Exterior, vol. 56, núm. 10, octubre 2006, pp. 843-853.

Blair, Roger, y Kenny Lawrence, Microeconomía con aplicaciones a la empresa, McGraw-Hill, México, 1982.

Borch, Odd-Jarl et al., "Competitive strategy and the capabilities of micro firms in the food sector: the potential for farm-based entrepreneurship", Nordland Research Institute, Bodo, Noruega, pp. 1-45, 1999.
Cabral, Luis, Introduction to Industrial Organization, Cambridge, Massachusetts, 2000.

Capozza, Dennis y Van Robert, "Product differentiation and the consistency of monopolistic competition: A spatial perspective", en Journal of Economic Literature, vol. xxxI, núms. 1/2, septiembrediciembre, 1982, pp. 27-39.

Chamberlin, Edwars, The theory of monopolistic competition, Cambridge, Harvard University Press, 1933. 
Clarke, Roger, Economía Industrial, Ed. Colegio de Economistas de Madrid y Celestes Ediciones, Madrid, 2000.

Consejo Estatal de Población de Baja California (CONEPO), La marginalidad en Baja California, Secretaría General de Gobierno, Baja California, México, 1995.

Consejo Nacional de Población (CONAPO), "Índices de Marginación 2000", México, 2001, en www.conapo.gob. $m x$

Demsetz, Harold, "The nature of equilibrium in monopolistic competition", en Journal of Political Economy, vol. 67, febrero, 1959, pp. 623-641.

Hall, Robert, "A framework linking intangible resources and capabilities to sustainable competitive advantage", en Strategic Management Journal, núm. 14, 1993, pp. 607-618.

Hayek, F.A, "The use of knowledge in society", en The American Economic Review, vol. xxxv, núm. 4, 1945, pp. 519-530.

Huergo, Elena, "El diagnóstico de poder de mercado en economía industrial: Una revisión de la literatura empírica española del siglo xx", documento de trabajo 200113, Biblioteca de la Facultad de CCEE y Empresariales UCM y PIE-Fundación Empresa, 2001, pública, en www.ucm.es/BUCM/ceel

Instituto Nacional de Estadística, Geografía e Informática (INEGI), en www.inegi.gob.mx

Kirzner, Israel, "Entrepreneurial discovery and the competitive market process: An Austrian Approach", en Journal of Economic Literature, vol. xxxv, marzo, 1997, pp. 60-85.

Lerner, A., "The concept of monopoly and the measurement of monopoly power", en The Review of Economic Studies, 1(3), junio 1934, pp. 157-175.

Mungaray, Alejandro, Natanael Ramírez y Texis Michelle, "Estructura de mercado y maximización de beneficios en las microempresas", en Comercio Exterior, vol. 55, núm 4, abril, México, 2005, pp. 316-321.
Ocegueda, Juan, “Análisis kaldoriano del crecimiento económico de los estados de México, 1980-2000”, en Comercio Exterior, vol. 53, núm 11, noviembre, México, 2003, pp. 1024-1034.

Palacio, Juan Ignacio, "Política industrial activa para microempresas en el funcionamiento de los mercados. Una perspectiva española", en A. Mungaray; J.I. Palacio y C. Ruiz Duran (coordinadores), Potencial de la vinculación universitaria para una política microempresarial. Una perspectiva española, MA Porrua, México, 2002.

Robinson, Joan, "What is perfect competition?", en The Quarterly Journal of Economics, vol. 49, núm 1, noviembre, 1934, pp. 104-120.

Romer, Paul, "The origins of endogenous growth", en Journal of Economic Perspectives, vol. 8, núm. 1, invierno, 1994, pp. 3-22.

Schumpeter, Joseph, "The creative response in economic history", en The Journal of Economic History, vol. viI, núm. 2, noviembre, 1947 , pp. 149-159.

Sistema de Consulta para la Información Censal por Colonias (SCINCE), V.2, INEGI, 2000.

Sistema de Información Empresarial Mexicano (SIEM), en www.siem.gob.mx

Solow, Robert, "A contribution to the theory of economic growth", en Quarterly Journal of Economics, vol. 70, núm. 1, febrero, 1956, pp. 65-94.

Tregaer, A., "Regional specialty foods in the UK: Consumer perceptions and producer activities", en Borch (editor), "Small-scale food production in a Nordic context. Proceedings of the first Nordic workshop on regional small scale food production", Nordland Research Institute, Bodo, 1998.

White, Horace, "A review of monopolistic competition and imperfect competition theories", en The American Economic Review, vol. 26, núm 4, diciembre, 1936, pp. 637-649. 